\title{
Our pale blue dot - are there other homes for us in our galaxy?
}

Corresponding author: Robert Walker (robert@robertinventor.com)

\section{Preprint DOI $\underline{10.31219 / o s f . i o / 634 m w}$}

Please note this article is not yet peer reviewed and is currently in the process of development. [Figures need permission.]

Abstract

Introduction

Can other planets be 'home from home'?

Are there 'homes from home' around other stars?

Do we eradicate incompatible biospheres?

Biosphere collisions on Mars

Finding a way forward with mutual respect

Exponential growth is not inevitable

Backup seed bank and library on the Moon

Expanding into the galaxy in habitat bubbles

Vulnerability of galaxy to self replicators - with minds (ourselves)

Are we first?

Sustainability solutions to Fermi's paradox

Where are all the explorers?

Combining several solutions to the Fermi paradox in one scenario

Other factors that could lead to future civilizations becoming far more abundant than present day ones

Transforming a potential invasive weed in our galaxy into a beautiful flower of a civilization

References 


\begin{abstract}
Carl Sagan proposed that aggressive galactic imperialism is intrinsically unstable to the point that civilizations disposed that way never spread far through the galaxy. He suggested that by the time civilizations advance as far as interstellar exploration they are pre-adapted to mutual respect. We introduce the idea of galaxy protection as an extension of planetary protection, that advanced civilizations need to develop ways to live sustainably on their planet, as we are doing, and that in the process they also develop an understanding of the need to protect their planets and their entire galaxy from themselves to explore it sustainably with minimal impact on other civilizations and on themselves. Development of attitudes of sustainability and planetary and galaxy protection may be part of an inevitable progression of advanced civilizations as they mature.
\end{abstract}

\title{
Introduction
}

Our pale blue dot is our natural home. Carl Sagan wrote (Sagan,1997)

\section{...There is nowhere else, at least in the near future, to which our species could migrate. Visit, yes. Settle, not yet. Like it or not, for the moment, the Earth is where we make our stand.}

There may be other life in our solar system. Perhaps primitive sponges live in thin films of salty water on Mars (Stamenković, 2018). Perhaps creatures like cuttlefish find a home below the icy surface of Europa, where hydrogen may vent from 25 kilometers deep cracks into an oxygen rich ocean (Vance, 2016).

Yet his words still apply. These are not homes for humans. Ours need oxygen and water, scrubbed of carbon dioxide. Tiny bubbles of our pale blue dot wherever we travel.

Here is the historic Voyager spacecraft photograph of our pale blue dot:. 


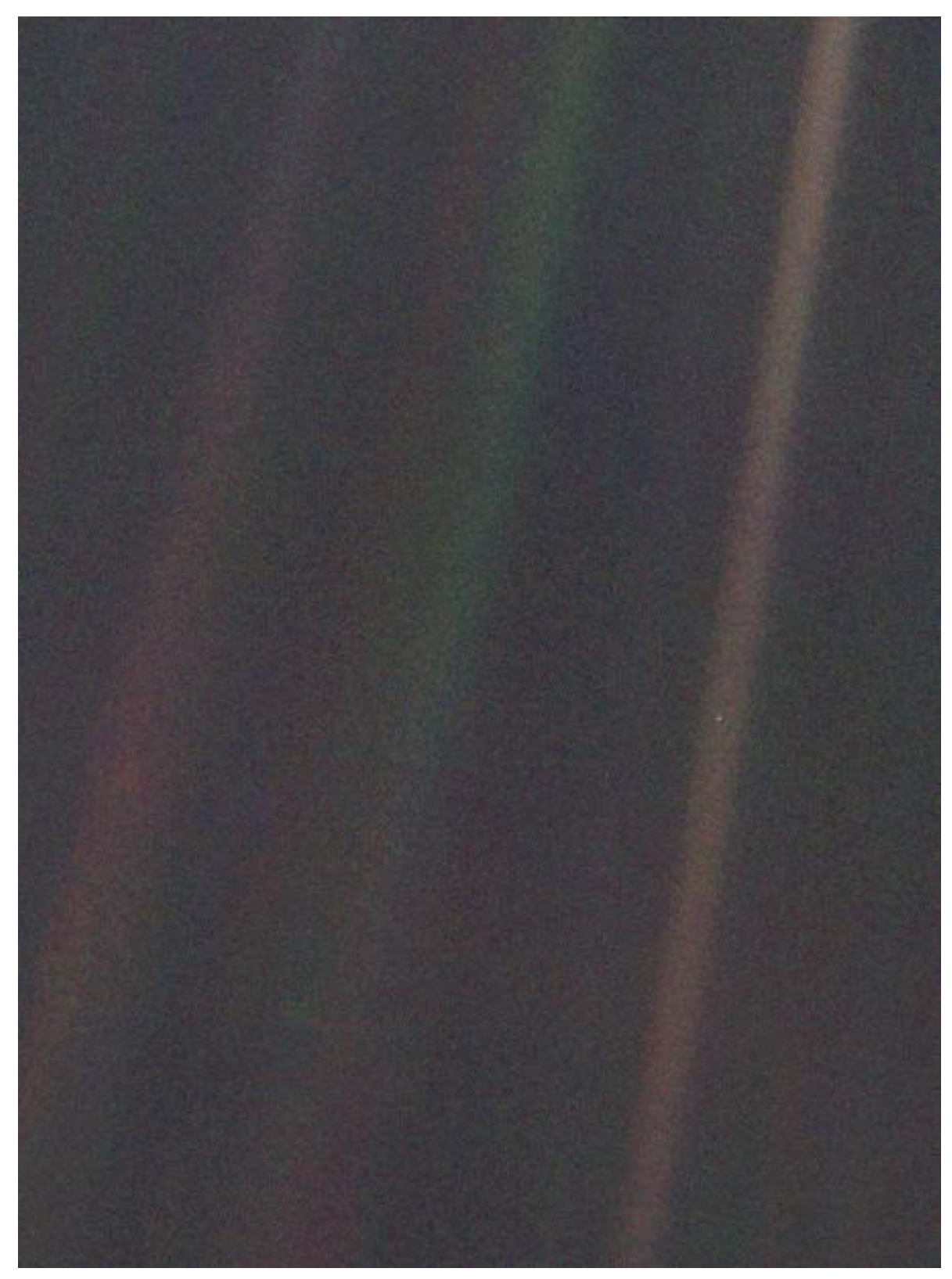

"To me, it underscores our responsibility to deal more kindly and compassionately with one another and to preserve and cherish that pale blue dot, the only home we've ever known."

\section{(Sagan, 1994)}

The bands are image artefacts, pointing the camera close to the sun.

This is a remastered version using modern image processing techniques. It still preserves the bands in the image: 
Nasa 're-masters' classic 'Pale Blue Dot' image of Earth 
Look at this remote island with eyes of a space colonist:

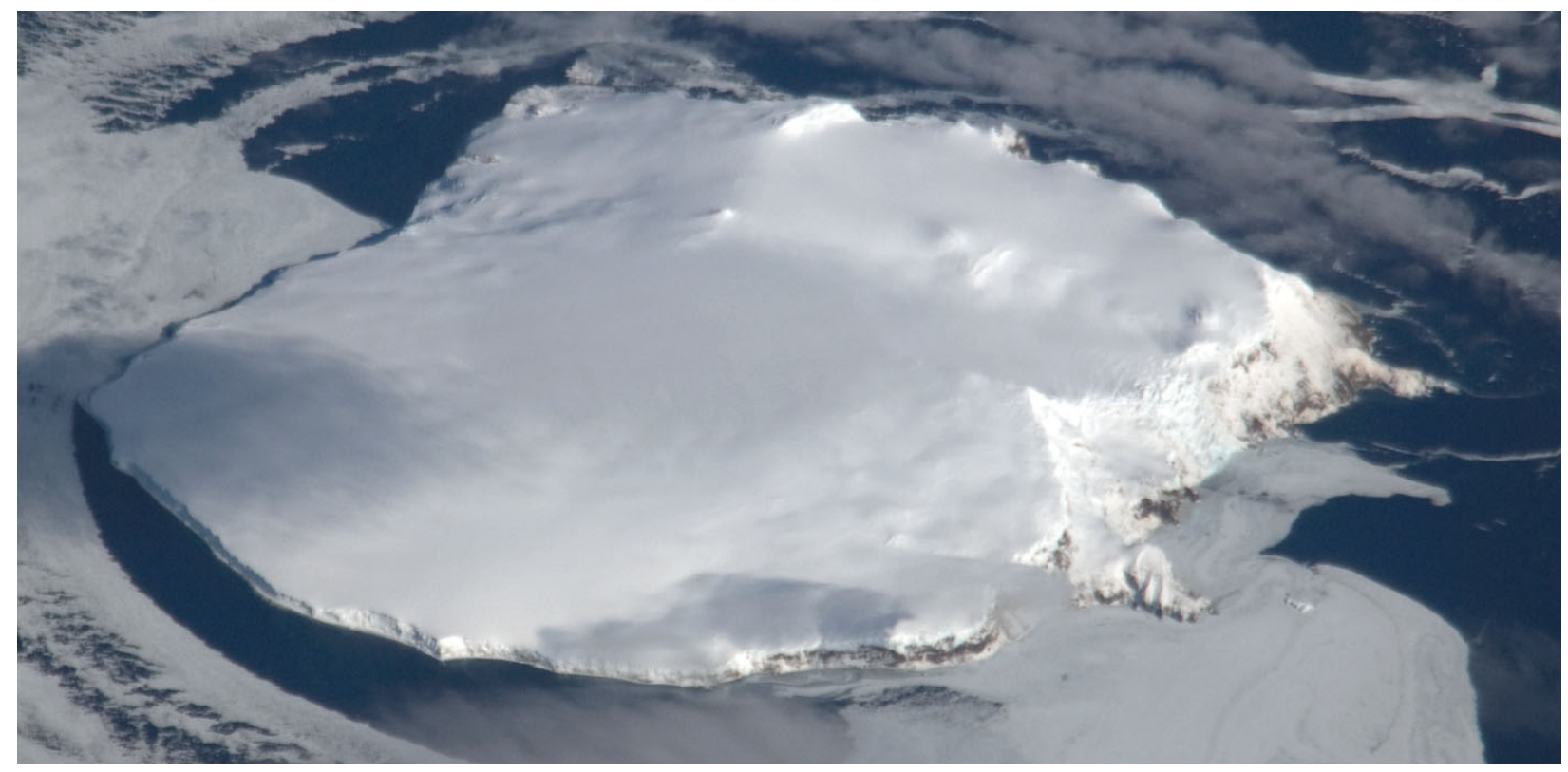

Bouvet island from the ISS. 1,600 kilometers from the nearest land, uninhabited, except by mosses, lichens, fur seals and penguins (NPI, 2016)

It has air for plants, and to breathe! Abundant ice! Seawater! Atmosphere to protect from solar storms, cosmic rays and small meteorites! It's a resource filled paradise.

Some feel we should set up a colony on another planet to ensure survival. Yet we have solved many problems already. Seen with eyes of a space colonist, life is so easy here.

Instead, let's preserve and cherish our pale blue dot, as our top priority. Let's open to a future of vast promise, living sustainably as socially responsible galactic citizens.

\section{Can other planets be 'home from home'?}

Could Earth become so uninhabitable, that other planets or moons become more attractive than ours as a place to live?

Let's look at some scenarios (Bostrom, 2002) (cite, date)

- There is no risk of runaway greenhouse warming to a hot house Venus. Earth has only a tenth of the carbon based fuel reserves needed (Goldblatt, 2013). The runaway methane "clathrate gun" is also disproved (Ruppel, 2017) (Knies, 2018). 
- For nuclear war, Nagasaki and Hiroshima are thriving modern cities, and much of the southern hemisphere is nuclear free. As for nuclear winter, 'soot from Kuwaiti oil fires did not rise as its models predicted (Sagan, 1995). A few persist with the idea but it is generally regarded as disproved (Seits, 2011).

- Supernovae and gamma ray bursts are only possible for rare types of star. We know our neighbourhood well now. They can't happen for thousands of years (Plait. 2010) (Gowanlock, 2016) (Walker, 2016).

- For synthetic and extraterrestrial life, we now have strong environmental regulations (Race, 1996). We'll see this could preclude rapid colonization if it risks returning microbes from Mars that harm Earth's environment.

- As for technological issues, the highest tech society ever conceived, set up in a rush in space, may create as many problems as it solves.

I have found only one scenario in the literature that makes Mars more attractive than Earth, the remote possibility of an impact by the large comet Swift Tuttle, in 4479 (Chambers, 1995). It's a chance in a million, and Earth hasn't been hit by anything so devastating since the last remnants of solar system formation hit it over three billion years ago. But let's see if a Mars colony would help.

Life in the deep sea would not notice Swift Tuttle, but surface layers of oceans would boil, evaporating an average of perhaps a meter of water (Walker, 2018a).

A young Mars colony would not last long without supplies from Earth. It's "atmosphere" is so thin, your lungs can't absorb oxygen without a pressure suit. On Earth, even if photosynthesis gets "switched off" for decades, the oxygen lasts 2,500 years before it drops to $60 \%$ (Walker. 2018b).

For far less cost, we can divert a large comet, triggering its jets to change its orbit [cite date]. Even for Swift-Tuttle, it's best to protect Earth directly.

There are places in our solar system that only need scuba gear or rebreathers, and drysuits, Saturn's moon Titan for one with its thick atmosphere (Wohlforth, 2017) 


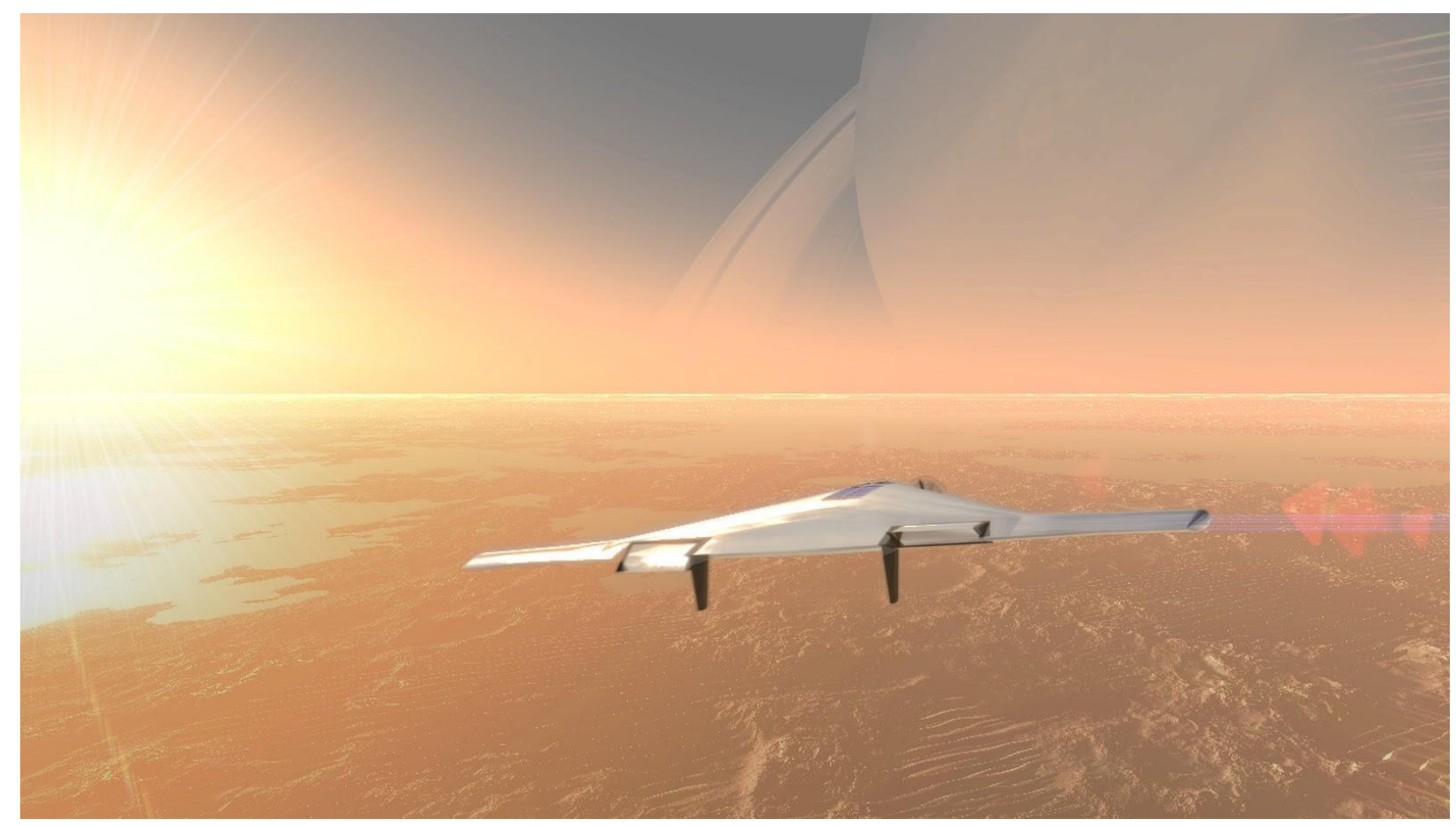

T-LEAF inflatable aerostat exploring Titan (Ross, 2015)

It's colder than dry ice, but this makes dry suits more insulating. Add heaters for hands and feet, and you are ready for a Titan expedition.

You could also use a drysuit or wetsuit in an artificial impact lake on Jupiter's moon Callisto. Venus cloud tops are similar in temperature and pressure to Earth. All you need is an acid resistant haz suit with a rebreather to venture outside your cloud tops aerostat.

However, although Jacques Cousteau and others attempted to found seabed colonies, none lasted long (Kaji-O'Grady, 2005), Titan, Callisto, or the Venusian clouds, like the seabed, are still "no place like home".

\section{Are there 'homes from home' around other stars?}

Our generations' great grandchildren may set off, perhaps using laser propelled light sails, and arrive at nearby stars after decades of travel.

There may be plants on some planets, and oxygen breathing animals (or their equivalent). If so, we could breathe the air, unless it has toxic gases from volcanoes or alien lifeforms. We could eat salt, simple sugars perhaps, and drink water. But what about the alien life? 
The food, based on an alien biochemistry, is likely to miss essential amino acids (MedlinePlus), replaced by any of 4,000 biologically reasonable alternatives according to one computer search (Meringer, 2013). Not nutritious, perhaps poisonous.

As our biospheres collide, our microbes will mingle. Even with friendly competition, how would you fare if half the microbes in your gut used an alien biochemistry? How would other Earth animals, or plants fare?

Perhaps in some ecosystems our biosphere predominates and in others, the alien one does, depending on temperature, salinity, aridity and so on. Perhaps sometimes one overwhelms the other everywhere.

We can also be harmed directly. Legionnaires' disease attacks our lungs in the same way it infects microbial biofilms. We have defences against Earth biology. We may be defenseless against the unfamiliar biochemistry of a disease of extraterrestrial biofilms. Joshua Lederberg (microbial geneticist) put it like this:

\section{If Martian microorganisms ever make it here, will they be totally mystified and defeated by terrestrial metabolism, perhaps even before they challenge immune defenses? Or will they have a field day in light of our own total naivete in dealing with their "aggressins"? (Lederberg, 1999)}

The physicist Claudius Gros looks at interpenetrating biospheres in his paper on a "Genesis project" to establish ecosystems on briefly habitable exoplanets. He assumes as a general principle in biology that defense mechanisms evolve only when the threat is actually present.

In his best case, some rapidly reproducing microscopic creatures survive. In his worst case, all that's left of a once magnificent biosphere is a mush of microbes (Gros, 2016).

\section{Do we eradicate incompatible biospheres?}

So what do we do if we find no other compatible biospheres? We could just take over. Alien life at an early evolutionary stage may be so vulnerable we don't have to do anything except introduce Earth life. Or prospective colonists could redirect comets to sterilize a planet.

This would be the ultimate in what Carl Sagan calls "galactic imperialism". Is there some more friendly way of starting a galactic civilization?

How else do we handle biosphere collisions?

\section{Biosphere collisions on Mars}


We don't know if there is life on Mars, but there are now many proposed habitats. Recent research suggests its brines might be oxygen rich, the coldest perhaps with enough for simple animals such as sponges (Stamenković, 2018).

Some Mars colonization enthusiasts assure us this life (if it exists) must be distant cousins, exchanged on meteorites. But impacts occur only every million years, and they send rocks from its high uplands, where air is thinner, and at least three meters below the ground. Transfer may be possible but there is no proof of this yet (NRC 2009). Many Earth microbes probably couldn't survive transfer to Earth (Cockel, 2008), so would Mars life?

Early Mars probably had seas, but there is no certainty it happened then either. Then, there are as large obstacles in the other direction from Earth to Mars (Stöffler, 2007)

Distant cousins can be incompatible too. Generalist rabbits from Europe outcompete marsupials in Australia (Lees, 2008). Diatoms from the northern hemisphere invade New Zealand freshwater lakes, probably arriving on wet diving gear (Spaulding, 2010).

The safest way to start, for both us and any Martian life, is to explore with sterilized rovers, from Earth, then from Mars orbit (Oleson, 2013). Astronauts "teleport" virtually to avatar robots anywhere on Mars using immersive Virtual Reality. These can float, creep into tiny caves, drive great distances or stay in one place for months, using nothing but solar power.

Let's find out what is there first, and then decide whether to mix biospheres.

\section{Finding a way forward with mutual respect}

We seem to have avoided what Carl Sagan called (Sagan, 1983)

"the intrinsic instability of societies devoted to an aggressive galactic imperialism".

He suggested, for galactic civilizations:

“Civilizations that do not self-destruct are pre-adapted to live with other groups in mutual respect."

He continues

This adaptation must apply not only to the average state or individual, but, with very high precision, to every state and every individual within the civilization "because we are so newly faced with this difficult alternative, because we can so 
easily feel our own predispositions to territoriality and aggression, and because our culture provides so little encouragement to a planetary perspective, such an alternative organization, especially for a highly technological society, might seem to us at first unlikely. The required changes might take thousands of years or more, if the society does not destroy itself first. ...

Perhaps - although we consider this unlikely - very few societies succeed in such a program. In any case, the result is that the only societies long-lived enough to perform significant colonization of the Galaxy are precisely those least likely to engage in aggressive galactic imperialism.

...

Some subset of moderately advanced civilizations may be engaged in the exploration and colonization of other planetary systems; however their very existence makes it highly likely that their intentions are benign and their sensitivities about societies at our level of technological adolescence delicate.

Following Sagan's suggestion, there may be a filter that acts earlier to filter out the ones that are not sustainable in their civilization.

It would take a lot to make Earth uninhabitable to an alien species as adaptable to many habitats on their planet as humans. It would be easier for a technological society of rainforest frogs say, some creatures adapted to a very limited range of conditions.

Perhaps the non sustainable civilizations go extinct (if fragile like rainforest frogs) or constantly boom and bust, rebuilding new civilizations from the ashes of the old civilization on their home planet until they learn to become sustainable and then go interstellar.

It may be that civilizations by the time they are star-faring have reached the point where they realize it is important to be sustainable and that also they realize they need to protect the galaxy. Another thing that could lead to that is if advanced civilizations discover ways to extend their lifespans. A creature that will live for a million years may care much more about protecting the galaxy.

From experience on Earth, we have become longer lived, we have almost eradicated hunger, we have almost universal literacy and greatly improved respect for human rights, also animal rights and we are already protecting our planet from asteroids and comets with searches with our great telescopes and satellites. We can protect ourselves from diseases as never before, the plague is now a thing of the past with development of antibiotics, and smallpox is eradicated from the world and many countries no longer have endemic measles, or rubella. Malaria is eliminated from many countries.

Civilizations are likely to protect their planet from disasters such as asteroid impacts and increase survivability of the biosphere. We are a very young species and we have only just 
started on technology but already have a very strong orientation towards sustainability and working together to solve problems. Probably most alien species would be more likely to become less vulnerable and almost impossible to eradicate once they develop technology.

There are many other proposed solutions to the Fermi paradox. They don't need to be exclusive. Some might be natural stay at homes, or retreat to virtual worlds, not be the natural explorers we are or develop to a point where the exploration is in the mind, in science, in art or other creative things. These may be much more interesting challenges to them than the idea of a relentless colonization of every star system they can find.

There may be lots of civilizations but one is the parent civilization that arose first and it has developed a strong basis of planetary protection and galaxy protection, and that it educates young species as they arise. Or there might be some educational lesson such as a distant galaxy which has been trashed by unwise exponential growth that we will discover once we have somewhat better telescopes. There might be an extraterrestrial civilization broadcasting a galactic library in our galaxy or even another one that will make it all clear.

There may be many ways that sustainability and planetary and galaxy protection are supported in extraterrestrial civilizations.

Many of us, especially our young, are become scared of fake and exaggerated stories that Earth is "doomed" through such things as climate change, nuclear war, asteroids, or absurd mythological planets. The most vulnerable are often suicidal[own experience].

As we continue in space in the spirit of Apollo and the Outer Space Treaty, for the benefit of all mankind, we can see Earth with eyes of a galactic citizen. This may reflect back to help us find social solutions, and hope for our Earth too.

\section{Exponential growth is not inevitable}

[Need to add cites to this section]

Extrapolate forward and I don't think we are going to be an exploitative species trying to convert the entire galaxy into a home for our kind. 'That is a dangerous future for a species that tries it too, because they are essentially letting loose an uncontrolled self replicator on the galaxy.

Instead - we already see our population set to peak or level off before 2100 . The countries with highest standards of living have the lowest birth rate. A space colony would have the highest standard of living of all and so would be likely to have the lowest birth rates, well below replacement. 
This is something that became clear after Sagan wrote his essay. We won't need to fill the galaxy with our kind in order to satisfy a need for exponential growth if we don't have exponential growth. This also doesn't need to be enforced in any strict way, it happens naturally as an outcome of improved living conditions and greater access to resources.

It takes such a short time for a star faring civilization in exponential growth to fill a galaxy that it is almost impossible we discover an alien civilization currently in exponential growth phase. Unless they are in a perpetual boom then crash cycle and we spot them growing - which doesn't seem likely for an intelligent future civilization.

This suggests that alien civilizations also find their populations naturally level off like we do.

They may be almost impossible to spot because they are so advanced that they are $100 \%$ sustainable or close to it. So they emit almost no discernible signature and they likely have small populations. The total population may be only a few billion in the entire galaxy. Even if it is a trillion or a quadrillion we wouldn't notice them.

Earth could easily have a quadrillion people living sustainably in our own solar system in space colonies, there is far more habitable area for habitats made from materials in the asteroid belt and later kuiper belt and Oort cloud than there is on planetary surfaces. Even if we expand to a quadrillion people in the galaxy we don't need to leave our solar system and we could stay here through to the red giant phase and white dwarf and beyond. If our main population stays concentrated around our sun, and we only send explorers to other star systems, and if our technology becomes more and more sustainable, and efficient,and our population levels off, our future selves could be almost undetectable by a civilization at our current level of technology even just a few light years away.

There could be a hundred thousand alien civilizations like that, and if each one only occupies a few stars then each civilization could be in a volume of about a million stars. The nearest civilization would be hundreds of light years away typically and almost impossible to spot at our level of technology.

\section{Backup seed bank and library on the Moon}

And - yes - we can do a "backup" in space. William Burroughs and others proposed a seed bank on the Moon (Burrows, 2004). The eternal dark of polar craters is passively cooled to liquid nitrogen temperatures (Hayne, 2015). This increases lifetimes of many seeds tenfold (Walters, 2004). A time vault of life, it would be immune to political upheavals, climate change, all manner of disasters, and be there a thousand years later if needed. 
They also suggest a backup library of digitized knowledge interrogated via radio. We could add librarians, who oversee the seed bank and grow plants such as mangoes, whose seeds can't be cryopreserved.

Later we can back up ecosystems in lunar lava tube caves, eventually in space habitats too. In the low gravity theoretically these tubes are stable at up to five kilometers in diameter (Blair. 2017) and by radar observations, perhaps over $100 \mathrm{~km}$ long (Sood, 2016). They could also backup extraterrestrial biologies from Mars, Europa, Enceladus etc.

\section{Expanding into the galaxy in habitat bubbles}

If we proceed like this, what happens to hopes to explore, and find new homes in the galaxy? We can use habitat bubbles instead.

Asimov talked about fixation on planetary settlement as "planetary chauvinism" (Asimov, 1982). The asteroid belt between Earth and Mars has enough materials for a thousand times the land area of Earth reformed into large habitats, slowly revolving for artificial gravity (Heppenheimer, 1977) (Ashworth, 2012.).

We can build these habitats in a decade rather than the millennia for terraforming. If large enough, they may be almost self-sustaining, with the help of some technology, like a planetary biosphere in miniature. In the process we build up experience useful if we attempt to modify planets later.

It is then not a big step to create habitats throughout our solar system. Large thin film mirrors can focus enough sunlight to keep us warm, well lit, and supplied with electrical power, using ice dwarfs out to beyond Pluto (Johnson, 1977)

Once we have easy fusion power, we colonize our distant "Oort cloud" of icy bodies. This mingles with clouds of other stars, stepping stones into the galaxy.

We keep terrestrial planets pristine, yet colonists by the quadrillions orbit every star. Perhaps young civilizations will not know of us, until they wonder about the lights criss-crossing their skies from passing spaceships.

This would give us sustainable homes throughout the galaxy. But then - can we protect the galaxy from ourselves?

\section{Vulnerability of galaxy to self replicators - with minds (ourselves)}


We might explore safely with robotic self replicators (Sagan, 1983) (Freitas, 1983), Build one robot and a million years later their progeny orbits every star. Humans, however, can't be programmed with immutable safety laws.

Colonizing ships leapfrog to distant icy objects, by thousands, then millions. Adventurous colonists embark on long journeys to nearby stars. They spread at up to a tenth of the speed of light, perhaps halved by leapfrogging. The 100,000 light years span of our galaxy gets filled within two million years. A blink in geological time.

The problem is, exploitative colonists expand most rapidly. Sheep can lamb less than a year after birth, typically with two lambs (Sheep 101), and humans have given birth aged six (Snopes, 2015)

Populous colonists double every few years, or every year. With cloning and artificial wombs, they increase as rapidly as locusts, not necessarily highly intelligent, with nothing left to invent. Such a culture and biology wins the race to fill the galaxy. Even doubling every century our galaxy runs out of matter to make humans within 13,000 years (Walker, 2015)

This takes us to Fermi's question, why aren't extraterrestrials here already? It just takes one civilization to expand exponentially, and it should get everywhere, yet there are no passing spaceships and no traces of abandoned mining operations in our solar system (Webb, 2015).

It's no solution to say they make themselves extinct first. Our civilization has distributed knowledge, amongst mammals able to survive with minimal tools, anywhere from the Kalahari to the Arctic. Even the dinosaur era ending Chicxulub asteroid impact did not make warm blooded mammals extinct.

Life is even more robust. A few microscopic multicellular lifeforms can evolve back to intelligent creatures and a new civilization within a few hundred million years.

As for a galaxy spanning civilization, I suggest any collapse would leave colonies thousands of light years apart, who don't learn about it until millennia later. Remnants with a high birth rate soon recolonize the galaxy. So this is no solution to Fermi's paradox either.

Are we first, and if so, what should we do? Or if ET's already solved this, how did they?

\section{Are we first?}

[Need to convert links in this section into cites]

Someone has to be first. There will be some civilization that was the first one to develop in their galaxy and there will be some civilization that was first in the entire observed universe. They will 
look at their evolutionary history and they will assume everyone would evolve as fast as they do. So that could be us.

At first this seems unlikely, since there are many sun-like stars that were born billions of years before our sun. However, the entomologist and ecologist Alexei Sharov, and the mathematician and theoretical biologist Richard Gordon, decided to plot the increase of complexity of DNA against the time of origin of the lifeform. They found a way to ignore junk and duplicated DNA, so that they could count only what is essential to its genes. They found that as life increases in complexity, it follows a near straight line on this plot, through many different changes of structure of organism, from the prokaryotes, to the eukaryotes with nuclei, worms, fish, and mammals. It's a log plot so the straight line means that it always takes about the same amount of time for the complexity to double.

They traced the timeline back for the complexity of our genes, expecting it to cross the zero line at the time of origin of life, and found to their surprise that the zero line is nearly ten billion years ago. That's over twice the history of the Earth.

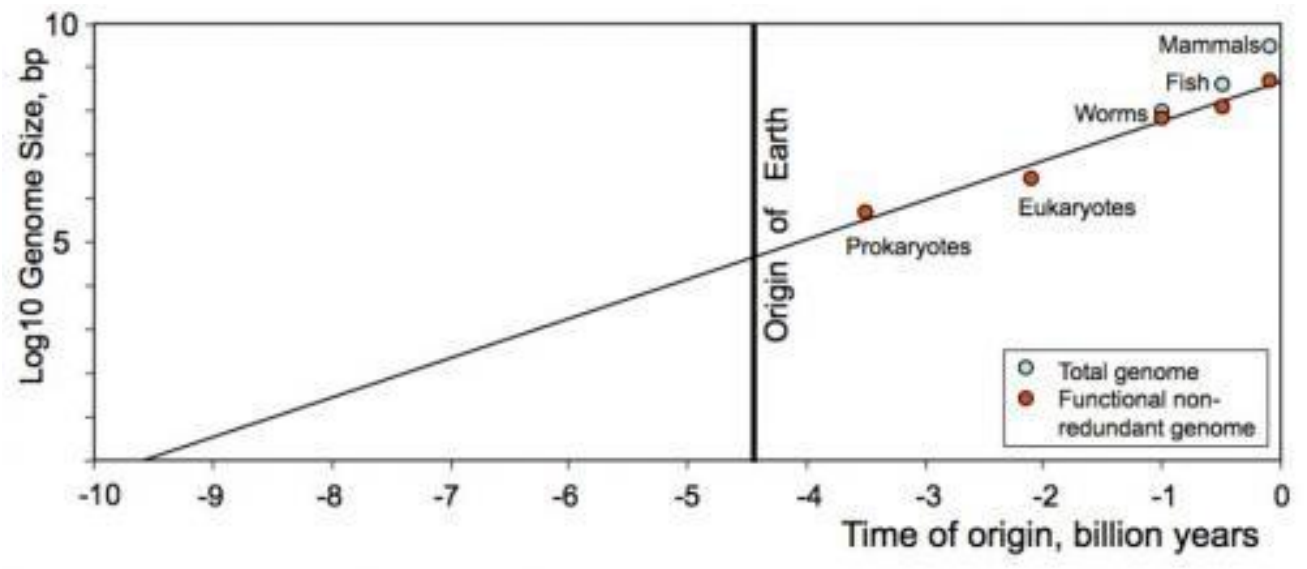

This diagram shows the complexity of the DNA as measured using the number of functional non redundant nucleotides. This is a better measure of the genetic complexity than the total length of its DNA. Some microbes have more DNA than a human being - much of that used for other purposes instead of genetic coding. This is the so-called $\underline{\mathrm{C}}$ Value Enigma. Measuring the DNA by functional non redundant nucleotides deals with that issue.

The graph is adapted from figure 1 of this paper which also explains in detail how it was derived.

Notice that the prokaryotes; the simplest primitive cell structures we know; are well over half way in complexity between the potential earliest forms of life and ourselves. Here, eukaryotes are cells with a nucleus to store the DNA, and prokaryotes don't have a separate nucleus. Mammals have around 3.2 billion base pairs or $3.2 \times 10^{9}$ The smallest 
prokaryote base pair has 500,000 base pairs (for Nanoarchaeum equitans and Mycoplasma genitalium) or $5 \times 10^{5}$.

Normally it's almost impossible for life to get from one star to another as they are so far apart. The problem was that after being ejected from another solar system, the rocks would pass through our solar system with high velocities, higher than the solar system escape velocity. Entering our solar system at perhaps six kilometers per second, due to the relative velocities of the stars, there would be almost no chance of capture.

However, recently Edward Belbruno and Amaya Moro-Martín reexamined this situation using Belbruno's new idea of "weak transfer", and also the idea of transfer while the stars are still in their birth nebula.

They found that our Sun could have exchanged life bearing rocks at least a hundred trillion times with its nearest neighbour in the cluster. Here they assume that the stars move at relative velocities as low as one kilometer per second as is normal in a young star forming region.

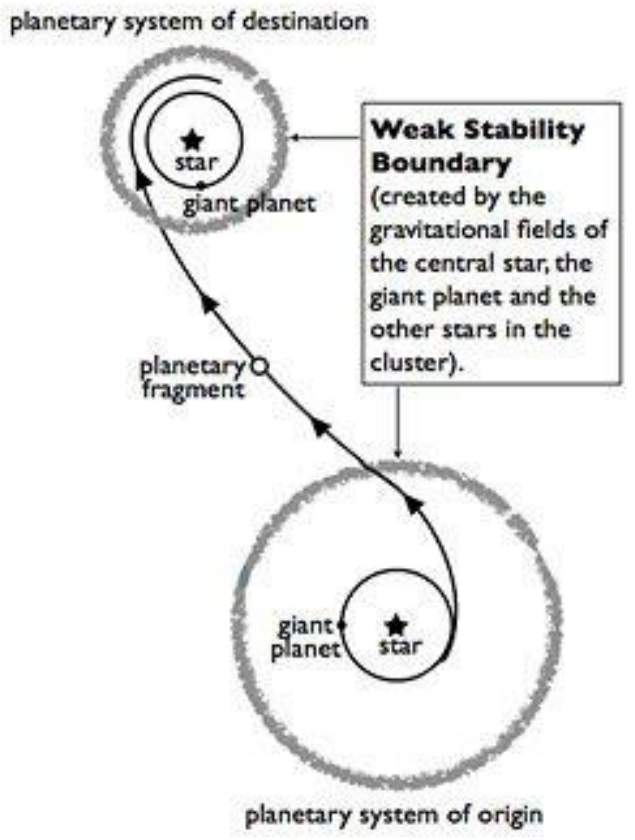

Shows how transfer of life was possible between two stars in the same cluster soon after the stars are born, with relative velocities between the stars of only of order one kilometer per second. It happens through the weak transfer, using the two weak stability regions shown, caused by the two stars themselves, other stars in the cluster and giant planets around both stars. 
Our solar system would have had perhaps 700 million years to do this before it got dispersed from the new cluster. It had liquid water already long before the end of that window of opportunity.

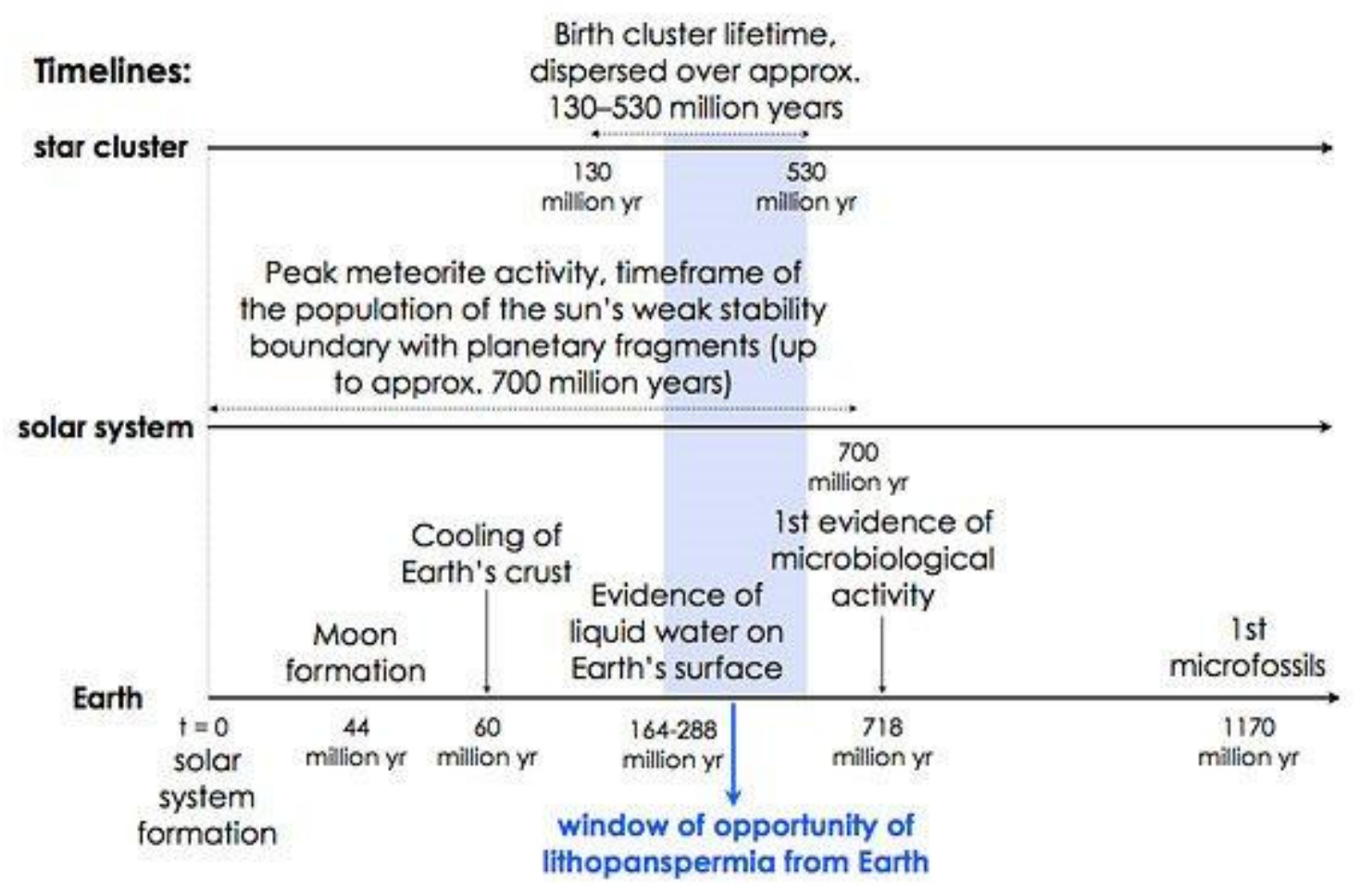

Table showing window of opportunity for panspermia in the early solar system sharing life with its sibling stars. Image by Amaya Moro Martin

the authors of another paper on this topic take this further, with a suggestion that life could be transferred from one cluster to another more easily than from star to star. With so much exchange of life between stars within a cluster, it would just need a single wandering star from a previous life bearing cluster to pass through a new star forming region, to infect all the stars in the new cluster. The stars from the previous cluster would soon spread throughout our galaxy and it just needs one of them to pass through a new star forming cluster to infect it.

This needn't be a sun like star. It could also have originated around a red dwarf star. This is by far the most abundant type of star in our galaxy, and it's also a very long lived type of star, lifetimes up to trillions of years and they have habitable regions that the planets could remain in for billions of years. 
Whether it was a red dwarf, an orange dwarf, a yellow dwarf like our Sun or some other star, only one of these stars is needed to seed the entire star forming region where our sun was born. Of course a star forming region is a far larger area to target than a single star, and if it originated in a nebula that was itself seeded with life, then there would be thousands of these life bearing stars around, and only one of those needs to pass through the next star forming region, for the life to propagate.

In the same way also, in the future, our Sun, or one of our Sun's siblings, such as HD162826, might infect stars in some future not yet born cluster, and so the process continues. In that way even if evolution of life is very difficult, it could still spread through much of a galaxy from a single origin. It would evolve further and further each time. The authors conclude:

" These results suggest that a young cluster is more likely to capture life from outside than to give rise to life spontaneously. Once seeded, the cluster provides an effective amplification mechanism to infect other members."

However it wouldn't be multicellular life, or even eukaryotes, because those came later on Earth. Our last common ancestor could also predate the prokaryotes, and be somewhat simpler than any form of life we know. Also it might or might not have photosynthesis which seems to have been a later development on Earth.

This scenario doesn't rule out the possibility of places in our solar system where we can study life evolving from scratch.

But it could be a way for our civilization to have its origins in ancient ancestors from stars that were born billions of years before our sun.

It then becomes much easier to argue that perhaps we are one of the first civilizations in our galaxy. Or we might be the first technological civilization in our galaxy.

For instance a civilization in the Europan ocean (if it is very habitable) would likely never develop our level of technology because they would have very limited access to heat and none to fire.

On our own planet, then most of the species nearly as intelligent as humans would not be able to develop technology easily if they had intelligence. If the ancient giant parrots in the Americas were intelligent and had a civilization, how would we know? We wouldn't even be able to detect a non technological civilization of giant squids in the deep sea right now today.

If we are first we have great responsibility to explore our galaxy sustainably - the future of the galaxy may depend on our decisions.

\section{Sustainability solutions to Fermi's paradox}


Carl Sagan proposed a slow colonization wave. He reasoned that you walk across Rome in hours, but it took far longer to build. A faster expanding civilization would be unable to sustain its infrastructure, and would collapse. Modeling this he came up with timescales so long a galactic civilization wouldn't get to us yet (Newman, 1978)

However, I suggest if sustainable Oort cloud colonies are possible, no external infrastructure is needed. Like self replicating robots, the expansion is close to the fastest safe speed for interstellar flight.

Seth Baum has also explored sustainability solutions. He discusses booms and crashes, civilizations that stay in their home star system, and ones that expand slowly and sustainably, understanding dangers of unsustainable expansion. Of the last he says (Baum, 2010).

"They are out there, but they are hard to find."

It's a good solution, but how could they be confident no splinter group embarks on exponential growth? Within a century or two humans may be able to set off through the galaxy. We have no way to prevent descendant 'aliens' returning, a few centuries later, perhaps with ideas stranger to us than the 'Borg' of Star Trek.

This can happen on a timescale similar to the time since the European colonization of the Americas. What we do now may influence that not so distant future.

Although we don't have a solution yet for galaxy protection, we can make a start by exploring and settling in a sustainable way, protecting our own planets. Once we have space settlement beyond Pluto, we are immune to almost anything, through into our sun's white dwarf phase, and trillions of years into its future.

We can travel through our galaxy as explorers, or send robotic self replicator envoys. If there is a way to colonize it sustainably, we are probably not first. Perhaps some day we meet our companion colonists. A galactic civilization with minimal impact on our galaxy may be hard to spot.

\section{Where are all the explorers?}

If there were, say, a million spacecraft exploring and they spent a century with each star system, a typical star system would expect to see an exploration starship every 100 * 100 billion / million $=10$ million years.

If there are a billion spacecraft exploring and they spend a thousand years on each star system, then it's once every 100,000 years, and so on. There could be large numbers of exploring 
starships out there and yet they just haven't got to us yet. And we don't know but it might be that many planets seem habitable.

Perhaps there are potentially civilization bearing planets around almost every star. We might not be such an obvious target as we think we are - until they detect our radar / radio / TV signals but if there are say 100,000 civilizations then there would be a million stars to every civilization, and the nearest one could be hundreds of light years away and we can expect a response some time a few centuries in the future.

Then - if they do explore and they have billions of exploring spacecraft, maybe they are here. They could have an equivalent of the Star Trek "prime directive" but much more strictly enforced. Perhaps they live out in the Oort cloud and rarely venture close to the sun so as not to disturb our civilization and only send undetectable robots here.

\section{Combining several solutions to the Fermi paradox in one scenario}

It's unlikely that any of the proposed solutions (Brin et al, 1983) (Cirkovic, 2009) (Webb, 2002) applies to all the civilizations. So let's try to sketch out a scenario that combines several of them in one description.

Our universe is clearly still very young. Most galaxies are still forming stars, and ours is one of the earliest of Earth-like planets born in the universe. Behroozi et al calculate that Earth formed earlier than $61 \%$ of all the Earth-like planets that will form in the Milky Way-Andromeda galaxy group (tracking forward to after the two galaxies collide). When Earth formed, only $8 \%$ of the halo of gas around galaxies in the universe had condensed to form stars, so $92 \%$ of the Earth-like planets in the galaxy are or will be younger than Earth (Behroozi et al, 2015).

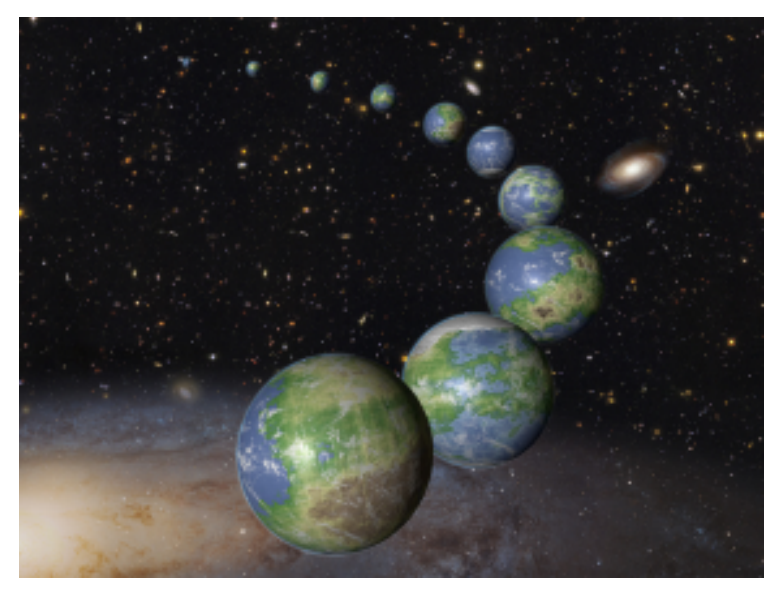

Artist's concept of future Earth-like planets in our young universe (Hubblesite, 2015) 
Berhoozi et al's best estimate for the number of Earth-like civilizations that will eventually form in our universe is $12.5 \times n$ where $\mathrm{n}$ is the number of civilizations that are in the universe at our current time since the Big Bang. This is based on their figure that only $8 \%$ of Earth-like planets in the universe were formed before Earth.

So -we may be first, but if not we are clearly amongst the earlier civilizations in our universe. In this scenario we assume life typically takes 10 billion years to evolve to our level of complexity and that our evolution started around another star.

Let's assume that, not counting us as a newcomer, there are 10,000 civilizations in our galaxy already, or one civilization for every 10 million stars.

Of those 10,000 civilizations, most are non technological. This would be unsurprising. Most of the other terrestrial species on Earth with intelligence approaching that of humans would not be able to develop technology, or not easily, if they had our level of intellectual comprehension of the world. Let's suppose one in ten of terrestrial civilizations develop technology.

Then, most of our planet is ocean and of the other proposed habitats in our solar system, the Enceladus and Europan habitats are oceans, Pluto may have a subsurface ocean - and there may be many worlds with subsurface oceans in the Oort cloud.

So let's suppose that one in ten planets have terrestrial civilizations, most are ocean dwelling and almost none of those have developed technology on their own because of the difficulty of generating fire in an ocean, though some may develop technology with assistance of terrestrial civilizations on the same planet.

So let's suppose that 9,900 of the civilizations are non technological. There are 100 technological civilizations in this scenario.

In that case then there is one technological civilization to every billion stars. Typically there are 100 non technological civilizations within that space.

The galaxy has about 4.1 billion sun-like stars (Patel, 2020) and as many as half may have Earth-like planets (Bryson et al, 2020). So there is one technological civilization for every 20 million Earth-like planets so far. That's being optimistic, their lower estimate is 300 million Earth-like planets, making it one technological civilization for every 3 million Earth-like planets.

Now for this scenario, let's suppose imperialism is rare, it does happen but most civilizations have evolved in their thinking to galaxy and planetary protection by the time they have developed the capability to colonize other stars, or are not interested in colonization.

So, let's suppose that - after the civilization has evolved through our present young phase for long enough to become star-faring, that typically there is one imperialist civilization to every 100 
non technological civilizations. So - let's suppose that our galaxy has one imperialistic civilization already.

Based on our supposition that only one in 100 civilizations is imperialistic, then it is unlikely that imperialistic civilization would be the first to develop. In this scenario we can expect one in a hundred galaxies had an imperialistic civilization arise first. We may see the effects of this early arising of imperialistic civilizations in distant galaxies, with the non sustainable booms we have looked at in this article, and busts of technology and warning messages broadcast to other galaxies from some of those worst affected, not to develop like that ourselves.

In this scenario we will also suppose that there is a lot still to discover about science and technology at our stage of development, but typically within 100,000 years there is nothing new to learn scientifically. By then, all the knowledge is found, or requires experiments that are not possible with the levels of energy possible within a galaxy and within the timescale available so far to carry out the experiments. This timescale may also be accelerated with galactic libraries sharing knowledge between civilizations so that new civilizations rapidly learn science that took thousands of years to develop. Perhaps later civilizations reach that point in just a few thousand years after they first encounter broadcasts by a galactic library. The libraries in this scenario could be set up by early evolving civilizations specifically to teach other civilizations how to protect themselves and their galaxy.

In this scenario then there are no unknowns by way of technology that adversaries could use against a civilization. Then - suppose that with the resources of a billion suns available, that a civilization can defend itself against all comers. This wasn't needed in our galaxy until the arising of the first imperialistic civilization - but the other extraterrestrials were fore-warned by messages from distant galaxies overrun by imperialistic civilizations, and built defences. The first imperialistic civilization expanded until it encountered the boundary with the nearest non imperialistic civilization and then could go no further except as ambassadors and explorers.

So then in this scenario, we are within the territory of one of the non imperialistic civilizations, that develop sustainably and with biorespect. In this scenario, that is not surprising as they are in the majority - and that's why no other extraterrestrials have taken over our planet or solar system and used its resources for themselves.

In this scenario, at present we are in the early period of the galaxy and universe and so civilizations so far are rare. The 9,900 non technological civilizations in our galaxy will eventually be contacted by technological civilizations but are hard to detect from a distance, and as life continues to evolve around the planets that don't have civilizations yet and as new stars and planets are born, eventually our galaxy will be a home to many more civilizations, including the ones that arise in the star burst during the merge of our galaxy with the Andromeda galaxy. 


\section{Other factors that could lead to future civilizations becoming far more abundant than present day ones}

Berhoozi et al's best estimate is only taking account of life that evolves around planets like ours. If civilizations can also develop in the Oort clouds of stars [needs cite], for instance, and if those civilizations evolve more slowly than ours, then in the future, once they have time to evolve, the number could be far more. The same also applies to young orange and red dwarfs. The smallest red dwarfs have life-spans of trillions of years, leaving plenty of time for civilizations to arise on them in the future, they are all still very young.

Also this is based on an assumption Earth evolved at a typical pace. But it could be that Earth evolved as much faster than is normal. There is some evidence suggests this, that the main steps in development of our life are roughly equally spaced which might suggest that each one happened as fast as is possible [needs cite].

Also - if evolution of life on Earth depended on previous evolution around other stars, then it could be that our galaxy has only just reached the point at which life anywhere in the galaxy has reached the level of complexity where civilizations can arise at all.

Life on Earth is then the result of 10 billion years of evolution and the most complex life on other Earth-like planets are also at a similar stage of evolution.

If it requires 10 billion years of evolution and we are a later development of life that evolved around another star that seeded our birth nebula, then maybe throughout the universe species are just starting to develop civilization. We would be amongst the first, - maybe we evolved unusually fast, but there may well be others. The first species would not be likely to be synchronized to within a thousand or even a million years but it could be that the earliest civilizations in each galaxy vary in their timing by only a few billion years, and that we are amongst the first few in our galaxy.

Another possibility here is that evolution of life is rare, and that most planets are not life bearing even after billions of years of an ocean with organics and chemistry and no life.

If so then most planets may depend on their birth cluster being seeded by another life bearing star system. If so, later Earth-like planets are more likely to be seeded by life than earlier ones, so the proportion of life bearing Earth-like planets increases with time.

It's also possible of course that star systems are seeded deliberately by other civilizations, or accidentally by them once they become star faring. This would also increase the proportion of life bearing Earth-like planets as the galaxy gets older. 
In that case then it could be that eventually we have a billion civilizations or more.

Indeed, if nearly all of the Earth like planets still to be born will have civilizations evolving from complex life seeded into their birth nebula from earlier planets, and on the optimistic assumption that there are 2 billion Earth-like planets so far, with $61 \%$ still to be formed, and assuming that the ones that don't develop technology are given technology by the ones that do, then we will eventually have over 5 billion technological civilizations around Earth-like planets.

There would be likely many more in the Oort clouds or around red and orange dwarfs etc.

But our universe is young and in the scenario sketched out in the previous section, though we are amongst the first, we are not the first in the universe.

\section{Transforming a potential invasive weed in our galaxy into a beautiful flower of a civilization}

The anthropologist Mary Russell, writes amusingly about our attempts to characterize what is unique about humans. She put it like this (Russell, 2012)

"Human beings are a dangerous, invasive weed species that has invented central heating, air conditioning, and food that can be stored for up to ten years, so not even a direct hit by an asteroid would likely make us extinct."

We would be especially devastating for any non technological civilizations we encounter. There may be millions of years old extraterrestrial civilizations, creatures similar to our dolphins, elephants, octopuses, and parrots. Advanced in all other respects, yet without technology, they would soon fall prey to the ineradicable weed of ourselves, youngest of technological aliens, who also then become a plague on themselves.

I suggest sensible ET's look at that possibility, and find a way to become a flower of the galaxy instead of an ineradicable weed. If they can't see a way, they stay at home until they can. As for the less sensible ET's, they destroy their own spaceflight capabilities in uncontrollable wars, until they develop sense. Ideas of sustainability and planetary and galaxy protection may be part of that social development towards galactic civilization that Carl Sagan talked about that he suggests happens inevitably before a civilization matures enough to be ready for interstellar exploration.

Let's be one of the civilizations in our galaxy and universe that flowers like a beautiful flower.

\section{References}


This material originated as the Galaxy protection - what about colonizing other star systems? section of my online Touch Mars? book (Walker, n.d.), several blog posts (Walker, 2015) (Walker, 2016) .(Walker, 2018a) (Walker, 2018b) (Walker, 2020a) (Walker, 2020b), and as an essay for my submission for the nine dots prize in 2019.

Ashworth, S., 2012. The Long-Term Growth Prospects for Planetary and Space Colonies. Journal of the British Interplanetary Society, 65, pp.200-217.

Asimov, 1982, interviewed by Bill Boggs, 35 minutes into this video

Baum, S.D., 2010. Is humanity doomed? Insights from astrobiology. Sustainability, 2(2), pp.591-603.

Behroozi, P. and Peeples, M.S., 2015. On the history and future of cosmic planet formation. Monthly Notices of the Royal Astronomical Society, 454(2), pp.1811-1817.

Blair, D.M., Chappaz, L., Sood, R., Milbury, C., Bobet, A., Melosh, H.J., Howell, K.C. and Freed, A.M., 2017. The structural stability of lunar lava tubes. Icarus, 282, pp.47-55 (popular article: BBC)

Board, S.S. and National Research Council, 2009. Assessment of planetary protection requirements for Mars sample return missions. National Academies Press, $\underline{\text { Chapter } 5 \text {, the }}$ Potential for large scale effects

Bostrom, N., 2002. Existential risks: Analyzing human extinction scenarios and related hazards, Journal of Evolution and Technology, Vol. 9, No. 1 (2002). (First version: 2001)

Brin, G.D., 1983. The great silence-The controversy concerning extraterrestrial intelligent life. Quarterly Journal of the Royal Astronomical Society, 24, pp.283-309.

Bryson, S., Kunimoto, M., Kopparapu, R.K., Coughlin, J.L., Borucki, W.J., Koch, D., Aguirre, V.S., Allen, C., Barentsen, G., Batalha, N.M. and Berger, T., 2020. The Occurrence of Rocky Habitable-zone Planets around Solar-like Stars from Kepler Data. The Astronomical Journal, 161(1), p.36.

Burrows, W., 2004, February. The survival imperative: using space to protect Earth. In 2004 Planetary Defense Conference: Protecting Earth from Asteroids (p. 1480).

Chambers, J.E., 1995. The long-term dynamical evolution of Comet Swift-Tuttle. Icarus, 114(2), pp.372-386.

Cirkovic, M.M., 2009. Fermi's paradox-The last challenge for copernicanism?. arXiv preprint arXiv:0907.3432. 
Cockell, C.S., 2008. The interplanetary exchange of photosynthesis. Origins of Life and Evolution of Biospheres, 38(1), pp.87-104.

Freitas, R.A., 1983. Extraterrestrial intelligence in the Solar System- Resolving the Fermi Paradox. British Interplanetary Society, Journal(Interstellar Studies), 36, pp.496-500.

Goldblatt, C., Robinson, T.D., Zahnle, K.J. and Crisp, D., 2013. Low simulated radiation limit for runaway greenhouse climates. Nature Geoscience, 6(8), p.661. See also New Scientist article about the research.

Gowanlock, M.G., 2016. Astrobiological effects of gamma-ray bursts in the Milky Way galaxy. The Astrophysical Journal, 832(1), p.38.

Gros, C., 2016. Developing ecospheres on transiently habitable planets: the genesis project. Astrophysics and Space Science, 361(10), p.324.

Hayne, P.O., Hendrix, A., Sefton-Nash, E., Siegler, M.A., Lucey, P.G., Retherford, K.D., Williams, J.P., Greenhagen, B.T. and Paige, D.A., 2015. Evidence for exposed water ice in the Moon's south polar regions from Lunar Reconnaissance Orbiter ultraviolet albedo and temperature measurements. Icarus, 255, pp.58-69 - see section 4.2. Temperatures of lunar south polar cold traps, figures 8 and 9 show some spots with max annual temperatures well below $60 \mathrm{~K}$ - liquid nitrogen boils at $77 \mathrm{~K}$

Heppenheimer, T.A., 1977. Colonies in space, by Heppenheimer, TA. Harrisburg, PA (USA): Stackpole, 224 p. $\underline{\text { Chapter } 2 \text { - Our Life in Space }}$

Hubblesite, 2015, Most Earth-Like Worlds Have Yet to Be Born. According to Theoretical Study

Johnson, R.D., 1977. Space settlements: A design study. nasa sp-413. NASA Special Publication, 413., chapter 7: View to the Future.

Kaji-O'Grady, S. and Raisbeck, P., 2005. Prototype cities in the sea. The Journal of Architecture, 10(4), pp.443-461.

Knies, J., Daszinnies, M., Plaza-Faverola, A., Chand, S., Sylta, Ø., Bünz, S., Johnson, J.E., Mattingsdal, R., Mienert, J. (2018): Modelling persistent methane seepage offshore western Svalbard since early Pleistocene. Marine and Petroleum Geology, 91, 800-811. blog

Lederberg, J., 1999. Paradoxes of the Host-Parasite Relationship. ASM News, 65(12).

Lees, A.C. and Bell, D.J., 2008. A conservation paradox for the 21st century: the European wild rabbit Oryctolagus cuniculus, an invasive alien and an endangered native species. Mammal 
Review, 38(4), pp.304-320. See page 309 ff "the ecology and impact of european rabbits as an invasive introduced species"

MedlinePlus, amino acids

Meringer, M., Cleaves, H.J. and Freeland, S.J., 2013. Beyond terrestrial biology: Charting the chemical universe of a-amino acid structures. Journal of chemical information and modeling, 53(11), pp.2851-2862. Popular article Mapping Amino Acids to Understand Life's Origins, NASA Astrobiology magazine

Newman, W.I. and Sagan, C., 1978. Galactic civilizations: Population dynamics and interstellar diffusion.

Norwegian Polar Institute, 2016, Bouvetøya (Bouvet Island), 27 January

Oleson, S.R., Landis, G.A., McGuire, M.L. and Schmidt, G.R., 2013. using telerobotic surface exploration from orbit.

Patel, N. 2020, Half the Milky Way's sun-like stars could be home to Earth-like planets, MIT technology review

Plait, 2010, No, a nearby supernova won't wipe us out - Bad Astronomy, Discover Magazine

Race, M.S., 1996, Planetary Protection, Legal Ambiguity, and the Decision Making Process for Mars Sample Return Adv. Space Res. vol 18 no 1/2 pp (1/2)345-(1/2)350

Ross F., Lee G., Polidan R., Sokol D., Sen B., Malmstrom R., Miller J.,, 2015 ,Lifting Entry and Atmospheric Flight (LEAF) Applications at Various Solar System Bodies, 12th International Planetary Probe Workshop, Cologne (see also 2016, Titan Lifting Entry \& Atmospheric Flight (T-LEAF) Concept Update)

Ruppel, C.D. and Kessler, J.D., 2017. The interaction of climate change and methane hydrates. Reviews of Geophysics, 55(1), pp.126-168 (Press release), USGS Gas Hydrates Project, 2017

Russell M.D., 2012, When nothing else matters, blog post for June 2, 2012

Sagan, C. and Newman, W.I., 1983. The solipsist approach to extraterrestrial intelligence.

Quarterly Journal of the Royal Astronomical Society, 24, p.113.

Sagan, C., 1994. A Pale blue dot. Public lecture, university of Cornell, October 13

Sagan, 1995. The Demon-Haunted World: Science as a Candle in the Dark (1st ed.). New York: Random House, page 257. 
Sagan, C., 1997. Pale blue dot: A vision of the human future in space. Random House Digital, Inc..

Seitz, Russell, 2011, Nuclear winter was and is debatable, Nature, 2011/07/06/online, 475, 37 https://doi.org/10.1038/475037b

Sheep 101, Mary had a little lamb ... or two, Sheep 101.info

Snopes, 2015, The World's Youngest Mother - 5 Years Old! Snopes Fact Check, 7 February

Sood, R., Chappaz, L., Melosh, H.J., Howell, K.C. and Milbury, C., 2016, March. Detection of buried empty lunar lava tubes using GRAIL gravity data. In Lunar and Planetary Science Conference (Vol. 47, p. 1509). (popular article: Universe Today)

Spaulding, S.A., Kilroy, C.A.T.H.Y. and Edlund, M.B., 2010. Diatoms as non-native species. The diatoms: applications for the environmental and earth sciences, pp.560-569.

Stamenković, V., Ward, L.M., Mischna, M. and Fischer, W.W., 2018. $\underline{\mathrm{O}}_{2}$ solubility in Martian near-surface environments and implications for aerobic life. Nature Geoscience, 11(12), p.905. see also (Press release)

Stöffler, D., Horneck, G., Ott, S., Hornemann, U., Cockell, C.S., Moeller, R., Meyer, C., de Vera, J.P., Fritz, J. and Artemieva, N.A., 2007. Experimental evidence for the potential impact ejection of viable microorganisms from Mars and Mars-like planets. Icarus, 186(2), pp.585-588. (See last para. for direction from Earth to Mars).

Vance, S.D., Hand, K.P. and Pappalardo, R.T., 2016. Geophysical controls of chemical disequilibria in Europa. Geophysical Research Letters, 43(10), pp.4871-4879,. press release, interview

Walters, C., Wheeler, L. and Stanwood, P.C., 2004. Longevity of cryogenically stored seeds. Cryobiology, 48(3), pp.229-244.

Walker, 2015, Why ET Populations Can't Continue To Expand For More Than A Few Millennia, Science 2.0 (blog post),

Walker, R.C., 2016, Debunked: A gamma ray burst could make humans extinct

Walker, R.C., 2018a, calculation in What will happen if Comet Swift-Tuttle strikes the Earth based on published mass of Swift-Tuttle, impact velocity and Figure 1 of Sleep, N.H., Zahnle, K.J., Kasting, J.F. and Morowitz, H.J., 1989. Annihilation of ecosystems by large asteroid impacts on the early Earth. Nature, 342(6246), p.139. 
Walker, R.C., 2018b calc in Debunked: If we cut down all the forests we will run out of oxygen to breathe - they are not the lungs of the planet in any literal sense based on figures in Buther, S. S., 1992, Global biogeochemical cycles, page 88 Academic Press, for photosynthesis, oxygen in atmosphere, and for sea level oxygen levels to remain above the $60 \%$ levels for the Tibetan plateau.

Walker, R.C., n.d. Touch Mars? See sections: Planetary protection for other stars and exoplanets and Galaxy protection - what about colonizing other star systems?

Walker, R.C., 2020a, Sustainability and planetary protection solutions to the Fermi paradox "where are all the aliens" - alternative to "great filter"

Walker, R.C., 2020b., No reason to fear aliens - sustainability, biorespoect, renewables - this is the way probably any forward looking ET goes and if they are in our solar system or galaxy already - they have minimal impact

Webb, S., 2015. If the Universe Is Teeming with Aliens... WHERE IS EVERYBODY?: Seventy-Five Solutions to the Fermi Paradox and the Problem of Extraterrestrial Life. Springer.

Wohlforth, C. and Hendrix, A.R., 2017. Beyond earth: our path to a new home in the planets. Vintage. 